\title{
LUMINOUS BLUE VARIABLE STARS IN THE MAGELLANIC CLOUDS
}

\author{
BERNHARD WOLF \\ Landessternwarte Heidelberg-Königstuhl D-6900 \\ Konigstuhl \\ Heidelberg 1 \\ Germany
}

\begin{abstract}
The main characteristics of the luminous blue variables (LBVs) in the MCs with S Dortype outbursts are reviewed. At quiescence they are luminous $O B$ supergiants occupying an inclined instability strip in the HRD. The most luminous LBVs are the hottest ones. During outburst, dense envelopes are expelled of equivalent spectral-type middle A to early F. LBVs occupy at maximum a vertical strip in the HRD at a temperature of about $8000 \mathrm{~K}$. The observed amplitudes in the visual range increase from $1 \mathrm{mag}$ (e.g. R71; $\mathrm{T}=14500 \mathrm{~K}$ at quiescence) to $2.5 \mathrm{mag}$ (e.g. $\mathrm{R} 127 ; \mathrm{T}=33000 \mathrm{~K}$ at quiescence). The existence of an amplitude-luminosity relation is suggested which can be used to derive extragalactic distances, if calibrated with the LBVs of the LMC. Whereas four LBVs are known to be members of the LMC, none is known in the SMC. This could be a consequence of the lower metal abundance in this galaxy.
\end{abstract}

